\title{
DNA scaffolds support stable and uniform peptide nanopores
}

\author{
Evan Spruijt ${ }^{1,2 *}$, Samuel E. Tusk ${ }^{3}$,Hagan Bayley ${ }^{1 *}$ \\ ${ }^{1}$ Chemistry Research Laboratory, University of Oxford, Oxford, OX1 3TA, UK \\ ${ }^{2}$ Present address: Institute for Molecules and Materials, Radboud University Nijmegen, Heyendaalseweg 135, 6525 \\ AJ Nijmegen, The Netherlands \\ ${ }^{3}$ Clarendon Laboratory, Department of Physics, University of Oxford, Oxford, OX1 3PU, UK \\ *Correspondence: hagan.bayley@chem.ox.ac.ukore.spruijt@science.ru.nl
}

The assembly of peptides into membrane-spanning nanopores might be promoted by scaffolds to pre-organize the structures. Such scaffolds could enable the construction of uniform pores of various sizes and pores with controlled permutations around a central axis. Here we show that DNA nanostructures can serve as scaffolds to arrange peptides derived from the natural polysaccharide transporter Wza to form uniform nanopores in planar lipid bilayers. Our ring-shaped DNA scaffold is assembled from short synthetic oligonucleotides that are connected to Wza peptides through flexible linkers. When scaffolded, the Wza peptides form conducting nanopores of which only octamers are stable and of uniform conductance. Removal of the DNA scaffold by cleavage of the linkers leads to rapid loss of the nanopores from the lipid bilayer, showing that the scaffold is essential for their stability. The DNA scaffold also adds functionality to the nanopores by enabling reversible and permanent binding of complementary tagged oligonucleotides near the nanopore entrance.

Membrane-spanning protein channels and pores play important roles in regulating transport across the cell membrane and in signalling and defence mechanisms ${ }^{1-3}$. Because they are versatile and amenable to engineering, nanopores have found application in single-molecule biosensors and sequencing technologies ${ }^{4,5}$. Larger nanopores are mostly $\beta$-barrels, many of which fold and insert into the outer membrane of gram-negative bacteria spontaneously or assisted by dedicated protein machinery ${ }^{6-8}$. Recently, a number of pores consisting of $\alpha$-helical peptides, including the polysaccharide transporter Wza and cytolysin A (ClyA), have been discovered, but their assembly is still poorly understood ${ }^{9-11}$. The ability to selectively bring together these and other peptides and stabilize their assemblies would both help address fundamental questions about membrane protein structure and function, and expand the utility of biological pores in biotechnology and synthetic biology. The building of robust and versatile scaffolds to realize 
these opportunities is therefore an important goal in nanotechnology. It is complicated by complex requirements for the structure and assembly of both the scaffold and the nanopore. The scaffold should dictate the size of the desired nanopore but must be sufficiently flexible to avoid trapping the peptides in an unwanted conformation. Moreover, the scaffold must not interfere with the lipid bilayer or obstruct the nanopore. Recently, we have shown that cyclodextrins can be used to template peptide nanopores ${ }^{12}$. These templates, however, cannot be used for large or heteromeric nanopores. In earlier work, four different peptide helices were tethered onto a flexible peptide backbone to form sodium channel analogues, but such peptide backbones may not be rigid enough to act as effective scaffolds for larger nanopores ${ }^{13-15}$.

To address this challenge, we have built a ring-shaped DNA scaffold composed only of short oligonucleotides. DNA has proven potential as a scaffold in nanotechnology and bioengineering ${ }^{16}$. Enzyme cascades have been spatially organized on DNA scaffolds ${ }^{17,18}$, although a proximity effect is still disputed ${ }^{19}$. Interacting membrane proteins have been coreconstituted in nanodiscs by using DNA scaffolding ${ }^{20}$. In bioengineering, DNA tetrahedra have been used for targeted siRNA delivery ${ }^{21}$, and logic-gated DNA nanorobots have been used to selectively interface with specific cell types in culture to induce cell-signaling ${ }^{22}$.

Here, we show that a ring-shaped DNA scaffold that is not anchored to the lipid bilaye $^{23,24}$, can bring together a defined number of 38-amino-acid amphiphilic peptides. The peptide is a variant of the $\mathrm{D} 4$ domain of the E. coli polysaccharide transporter $\mathrm{Wza}^{9,10}$. The scaffolded Wza peptides formed conducting nanopores of which only octamers are stable and of uniform conductance. DNA scaffolds with fewer than 8 peptides did not form stable conducting nanopores, whereas scaffolds with more than 8 peptides yielded nanopores with the same conductance as octamers. When the scaffold was removed after bilayer insertion, the pores were lost within minutes, demonstrating that the scaffold is essential for the stability of these nanopores. Finally, the scaffold can add functionality to the peptide nanopores by acting as a docking site for tagged oligos near the nanopore entrance.

\section{Design of a ring-shaped DNA scaffold from short oligos}

We designed a DNA ring with 12 arms as a scaffold for pore-forming peptides. The ring is composed of 12 unique 43-mer oligonucleotides (oligos) and has a diameter of $13.6 \mathrm{~nm}$ (Fig. 1a). Each oligo consists of four segments: the two central segments cover two helical turns and hybridize to form the ring, whereas the two outer segments contribute to each of two arms. Single unhybridized bases form hinges that separate the central segments in the ring from the outer segments and give flexibility to the arms. 


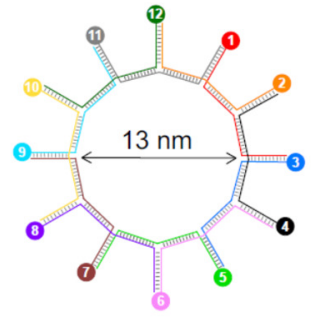

DNA scaffold

b

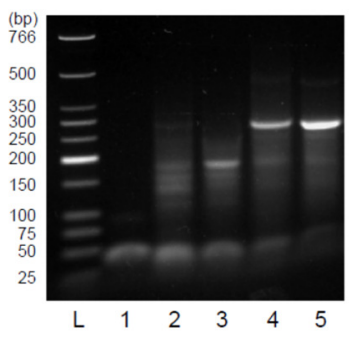

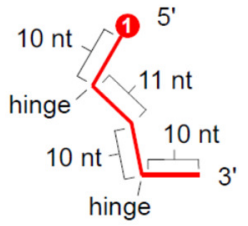

C

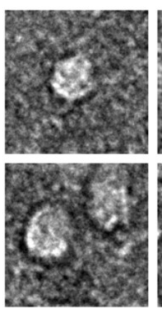

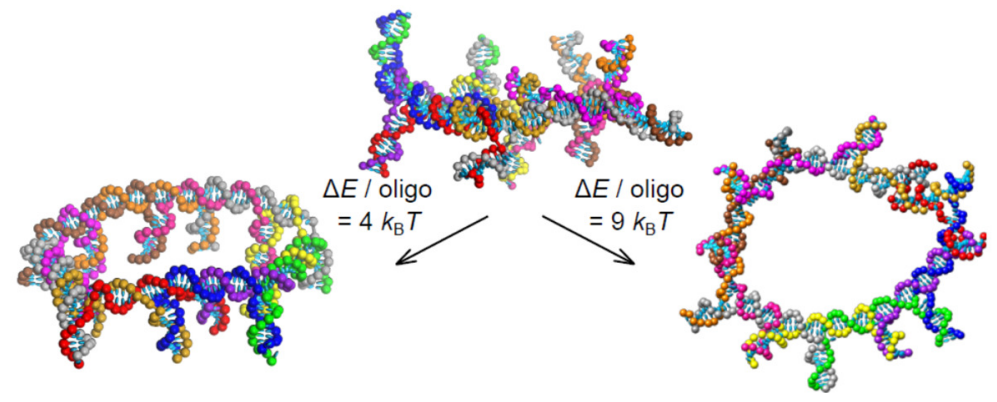

d

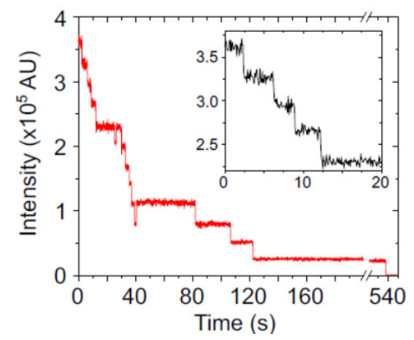

e

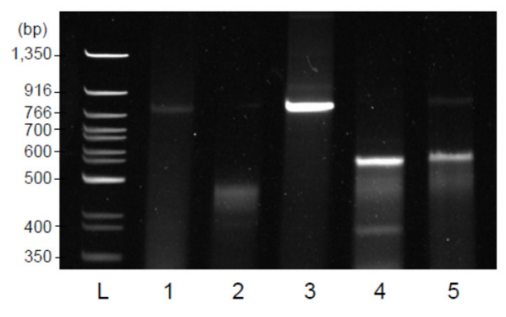

Figure 1 | Design, assembly and characterization of the DNA scaffolds for peptide nanopores.

a, Schematic of the DNA scaffold with oligos numbered at their 5' ends (left) and snapshots of the scaffold from coarse-grained molecular dynamics simulations (right) without constraints (top) and with arms forced down or outward (bottom). b, Scaffold annealing was analysed in a $2.5 \%$ agarose gel, stained with SYBR Gold. The gel shows an NEB low MW dsDNA ladder (L), for which the band sizes are indicated to the left of the gel, a single oligo (1), a mixture of 12 oligos without annealing (2), 11 oligos after annealing (3), 12 oligos after annealing in $100 \mathrm{mM}$ potassium phosphate, $\mathrm{pH} 7.2$ (4), and after annealing in $100 \mathrm{mM}$ potassium phosphate, $\mathrm{pH} 7.2$, with $0.5 \mathrm{M} \mathrm{NaCl}$ (5). c, Negatively-stained TEM images of DNA scaffolds annealed in $100 \mathrm{mM}$ Tris. $\mathrm{HCl}$, pH 7.2 (see Supplementary Methods for additional images). d, Stepwise photobleaching trace of a DNA scaffold formed from 12 Alexa-647modified oligos (see Supplementary Methods for additional traces). Inset: zoomed-in view of the first 20 s. e, Restriction digestion of the DNA scaffold, analysed in a 5\% polyacrylamide gel, stained with SYBR Gold. The gel shows an NEB 50 bp dsDNA ladder (L), for which the band sizes are indicated to the left of the gel, a mixture of 12 oligos without annealing (1), a mixture of 11 oligos after annealing (2), closed rings formed by annealing 12 oligos (3), annealed control construct identical to the RsaI restriction product (4), and closed rings incubated with RsaI in $100 \mathrm{mM}$ potassium phosphate, $\mathrm{pH} 7.2$ (5).

The balance between rigidity and flexibility is of critical importance for a supramolecular scaffold $^{25-28}$. Therefore, we performed coarse-grained molecular dynamics simulations of the scaffold in a solution of $0.5 \mathrm{M}$ ionic strength at $25^{\circ} \mathrm{C}$, in both the presence and absence of an external force. The scaffold is very stable with an average of 243 out of 246 base pairs formed. The ring-shaped backbone remains closed, while the unconstrained arms pivot around their attachment points, with a preference for alternating orientations of odd and even arms, above and below the ring (Movie S1). When a constant force of more than $9 \mathrm{pN}$ in a direction perpendicular to the plane of the ring is applied to the arms, all of them become oriented in the same direction (Fig. 1a, Movie S2). The free energy of the resulting structure is higher than the unconstrained structure by $4 k_{\mathrm{B}} T$ per oligo, but such a small energy difference is easily compensated for by a favourable interaction energy between residues on adjacent peptide $\alpha$-helices attached to the arms, or between those peptides and a lipid bilayer, both of which can reach $3 k_{\mathrm{B}} T$ per residue ${ }^{29}$. 
The DNA scaffold was assembled by mixing equal amounts of all 12 oligos in potassium phosphate buffered-saline, heating the mixture and cooling it to room temperature over $4 \mathrm{~h}$. Above $0.5 \mathrm{M}$ of monovalent salt, the scaffold formed with a yield of $90 \%$, as estimated by gel electrophoresis (Fig. 1b). If one oligo was missing from the mixture, or if annealing was omitted, the yield of assembly decreased to less than 30\%. In TEM images on negatively stained samples, the scaffolds were recognizable as ring-shaped objects with a diameter of $13.8 \pm 2.0 \mathrm{~nm}$, in the expected range, but the individual arms could not be distinguished (Fig. 1c and Fig. S1a). Class averaging of individual objects did not improve the resolution, probably because the scaffold is too flexible. Instead, we verified the scaffold stoichiometry by native mass spectrometry and stepwise photobleaching experiments. The mass of the intact scaffold is in agreement with the predicted combined mass of the 12 oligonucleotides (Fig. S2). Accordingly, photobleaching of scaffolds formed by 12 Alexa-647-modified oligos proceeded in a maximum of 12 steps (Fig. 1d and Figs. S3-S4). Finally, digestion with the restriction enzyme RsaI was used to establish that the scaffold is circular. Our design contains a single RsaI recognition site in the ring between arms 11 and 12. Incubation of the scaffold with RsaI resulted in opening of the ring yielding a linear fragment with 12 side chains that migrated faster in a polyacrylamide gel than the undigested scaffold and at the same rate as a control DNA construct designed to be identical to the restriction product (Fig. 1e and Fig. S1b).

\section{Scaffolding yields stable Wza peptide nanopores}

We tested the scaffolding capabilities of the DNA ring by attaching peptides with a propensity to form pores to the arms. The selected peptide CGG-cWza[T376R] (Fig. 2a; Wza peptide in short), is a variant of the consensus sequence of the Wza D4 domain (cWza) that does not form stable open pores by itself, because it contains two cationic residues near the $\mathrm{C}$ terminus (K375/R376) and lacks a stabilizing mutation, such as Y373C, near the C-terminal entrance of the pore ${ }^{12}$. Without scaffolding, this peptide produces only short-lived, noisy channels in planar lipid bilayers with an average open lifetime of $3.0 \mathrm{~s}$ at $+150 \mathrm{mV}$ and a unitary conductance of $0.32 \mathrm{nS}$ (Figs. S7-S13).

The peptide was attached to each of the 5'-amino-modified oligos at the N-terminal CysGly-Gly handle through a flexible linker $\left(\mathrm{SMPEG}_{2}\right)$. The 12 peptide-oligo conjugates were individually purified by HPLC, lyophilized, and analysed by ESI-MS (Fig. 2b and Table S1). We assembled DNA rings with various numbers of attached peptides at predefined positions (see Table S2 for a detailed list) around the ring by combining the desired peptide-conjugated oligos with unmodified oligos while keeping the stoichiometry fixed. Attached peptides decrease the electrophoretic mobility of both the oligos before annealing and the DNA rings after annealing 
a
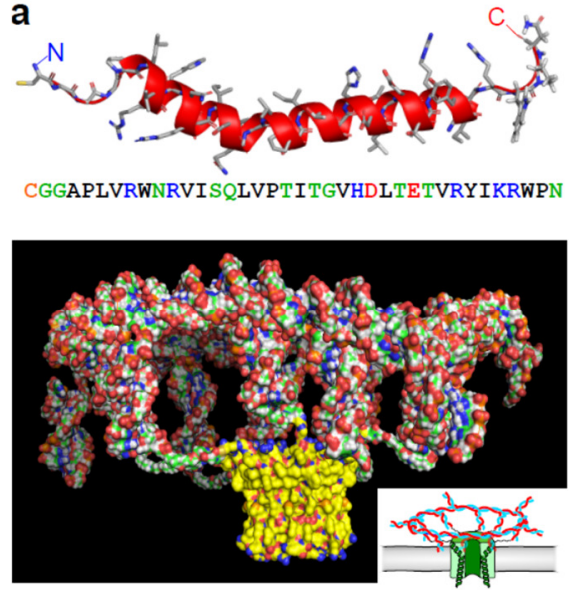

b
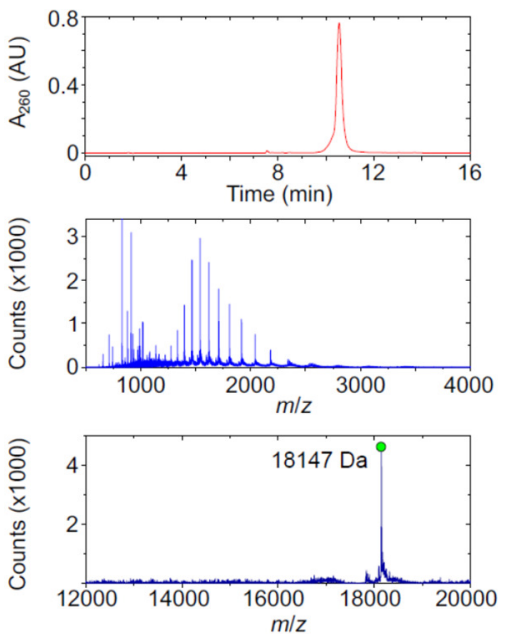

c

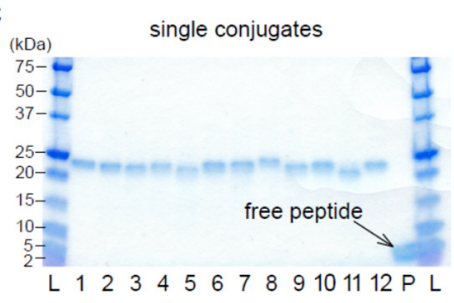

annealed hybrid rings

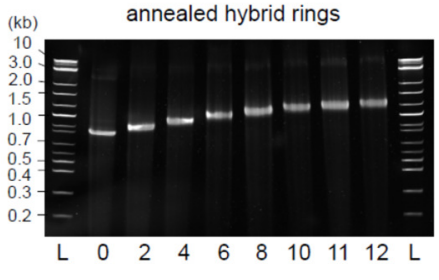

Figure 2 | Attachment of the Wza peptides to oligos and annealing of peptide-bearing DNA scaffolds. a, Sequence and structural model of CGG-cWza[T376R] (top) and a full-atom representation of a simulation snapshot (bottom) of 8 Wza peptides attached to the DNA scaffold from Fig. 1a (see Supplementary Data 1-3 for details). b, HPLC trace, ESI mass spectrum and maximum entropy deconvolution of the mass spectrum for the purified conjugate of Wza and oligo \#1 (see Supplementary Data 1). The deconvoluted spectrum shows a single peak with the expected mass of 18,147 Da. c, Polyacrylamide gel analysis of the purified peptide-oligo conjugates before and after annealing into rings. The top gel shows the individual peptide-oligo conjugates (1-12), run in an AnykD SDS-PAGE gel together with a Bio-Rad Precision Plus Dual Xtra protein marker (L), for which the band sizes are indicated to the left of the gel, and free Wza peptide $(\mathrm{P})$. The gel was stained with stained with InstantBlue. The bottom gel shows annealed DNA rings, with an increasing number of attached peptides (0 to 12$)$, run in a 5\% polyacrylamide gel without SDS, together with a NEB 2-log dsDNA ladder (L), for which the band sizes are indicated to the left of the gel. The gel was stained with SYBR Gold and the DNA detected by fluorescence.

and the number of attached peptides is readily estimated from the position of the band of the hybrid DNA-peptide ring in a polyacrylamide gel (Fig. 2c).

The pore-forming properties of the scaffolded Wza peptide were examined by electrical recording in planar lipid bilayers (Fig. 3a-d). When 8 peptides were attached to the scaffold we observed spontaneous insertion into 1,2-diphytanoyl-sn-glycero-3-phosphocholine (DPhPC) bilayers and formation of stable open pores at a scaffold concentration of $4 \mathrm{nM}$ and an applied potential between $-100 \mathrm{mV}$ and $-150 \mathrm{mV}$. In $72 \%$ of cases, pore formation occurred in two steps: first a noisy low-conductance state $(\mathrm{L}, 0.54 \pm 0.03 \mathrm{nS}$ at $-50 \mathrm{mV})$ was observed, which opened after several tens of seconds to a higher conductance state $(\mathrm{H}, 1.46 \pm 0.06 \mathrm{nS}$ at $-50 \mathrm{mV})$, characterized by a much lower noise (Fig. 3a). These insertions were sometimes preceded by a precursor state that we identified from bursts of transient spikes, similar to previous observations with the cWza consensus sequence ${ }^{12}$, and we occasionally observed switching between $\mathrm{L}$ and $\mathrm{H}$ for a brief period immediately after insertion. In the remaining $28 \%$ of cases, pore formation occurred either directly to the H-state (Fig. 3b) or after a noisy transition without reaching a stable intermediate conductance level (Fig. 3c). In all cases, the high conductance level that was eventually reached, was the same, as judged from the low current noise, the identical subconductance states and transient closures (Fig. S14), and the narrowly distributed mean 


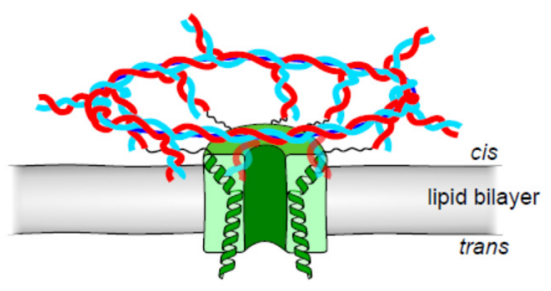

$\mathbf{a}$

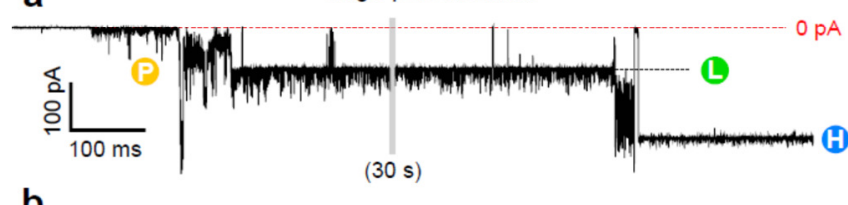

b

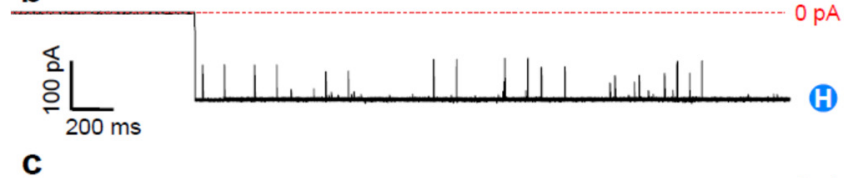

C
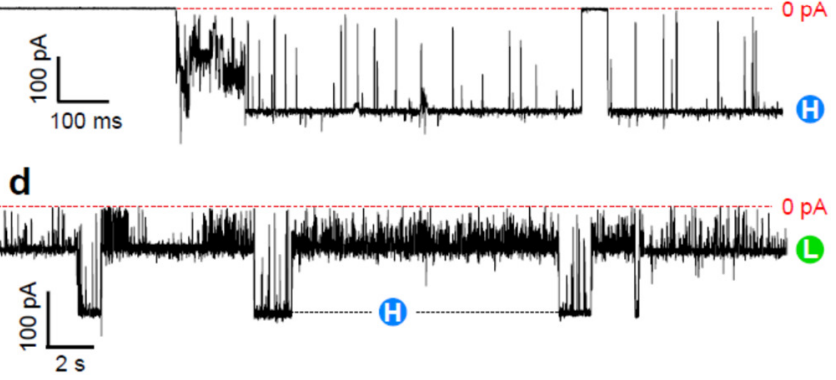

e
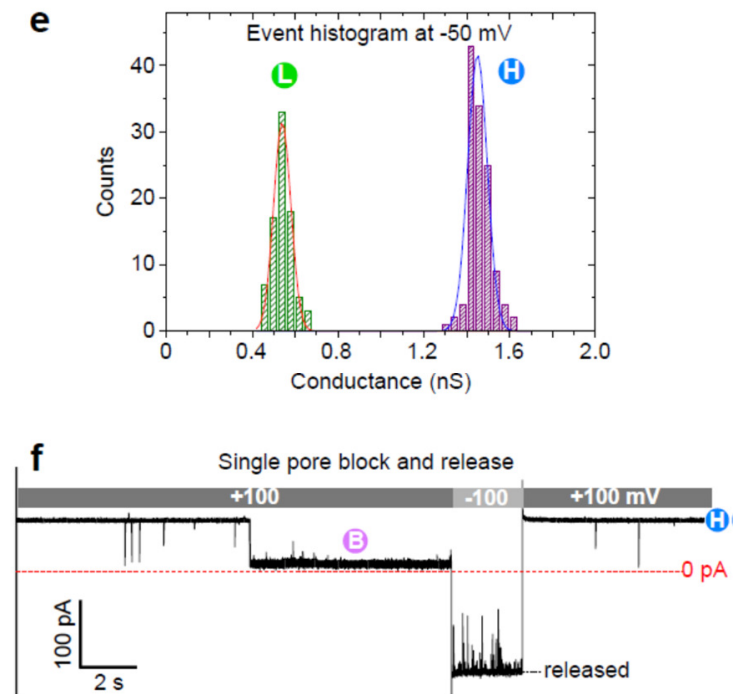

g

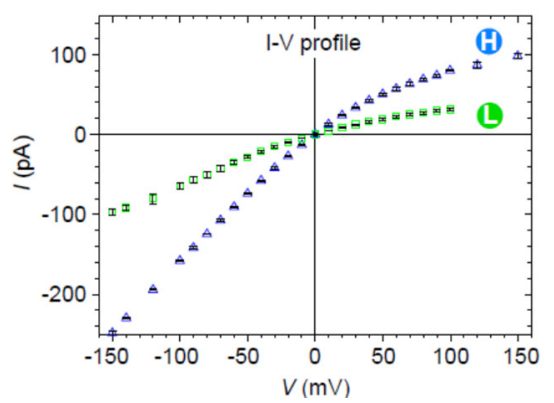

Figure 3 | Electrical properties of DNA-scaffolded octameric Wza nanopores.

a-d, Electrical recordings of the insertion of Wza nanopores formed from DNA scaffolds bearing 8 Wza peptides into planar DPhPC lipid bilayers. Insertions may occur via a precursor $(\mathrm{P})$ and an intermediate L-state (a, recorded at -150 $\mathrm{mV})$, without an intermediate state $(\mathbf{b}$, recorded at $-120 \mathrm{mV})$, or without reaching a uniform intermediate conductance level (c, recorded at $-150 \mathrm{mV}$ ). Switching between the intermediate L-state and the open pore H-state may occur briefly after insertion (d, recorded at $-120 \mathrm{mV}$ ). e, Event histogram of the electrical conductance values of 124 DNAscaffolded octameric Wza nanopores (see Table S2 for structural details), recorded at $-50 \mathrm{mV}$. f, Electrical recording of ssDNA-streptavidin (poly $(\mathrm{dC})_{50}$-biotin:streptavidin $=1: 3,200 \mathrm{nM}$ ) blocking $(\mathrm{B})$ and being released from a single DNA-scaffolded octameric Wza nanopore (21 blockades were observed with 3 nanopores). g, Averaged currentvoltage characteristics of nanopores formed by five different DNA-scaffolded octameric Wza nanopores in their Land $\mathrm{H}$-states. The error bars indicate one standard deviation.

conductance of $1.46 \pm 0.06 \mathrm{nS}$ (Fig. 3e), which is comparable to the variation found in natural protein nanopores, such as $\alpha$-haemolysin ${ }^{30}$. Once a high conductance state was reached, the scaffolded pores were stable and could be kept in an open state for at least an hour at applied potentials between $-100 \mathrm{mV}$ and $+100 \mathrm{mV}$ (Fig. S15).

The two observed levels ( $\mathrm{L}$ and $\mathrm{H}$ ) are different conductance states of the same pore. This is supported by the observed switching between the two levels (Fig. 3d), the fact that the ratio of the two conductance levels (2.7, Fig. 3e) is not an integer, and the observed single-step blockades (residual current $11 \pm 4 \%$ ) of the H-state by a streptavidin-bound biotinylated oligo (Fig. 3f). Although the two levels are states of the same pore, the current noise differs significantly between them. In the L-state, the coefficient of variation $(\mathrm{CV})$ of the current is $11 \%$ (at $-150 \mathrm{mV}$, filtered at $2 \mathrm{kHz}$ ), whereas it is only $3.1 \%$ in the $\mathrm{H}$-state, suggesting that the $\mathrm{H}$-state is a more static configuration. The H-state has a remarkably low noise compared to most nanopores formed 
from peptides ${ }^{31,32}$, but it is more noisy than some well-known natural protein nanopores, such as $\alpha$-haemolysin $(\mathrm{CV}<1 \%$ when filtered at $2 \mathrm{kHz})$. The scaffolded pores show significant rectification of the ionic current in both states (Fig. 3g), with $-\mathrm{I}_{+50} / \mathrm{I}_{-50}=1.51$ for the L-state and 1.42 for the H-state, which is most likely caused by the two cationic residues (K375/R376) near the C-terminal pore entrance (see Fig. S12 for data on mutant peptides). A more extensive study of different mutants would be required to confirm this.

To examine the possibility of scaffolding larger peptide nanopores, we annealed DNA rings with between 0 and 12 peptides and investigated their pore-forming properties in planar lipid bilayers (see Table S2 for details). We found that all scaffolds carrying at least 8 peptides led to the insertion of pores as shown in Fig. 3a-e with the same unitary conductance and rectification as the octamer. We observed some rare double and triple insertions (in 3\% of experiments) of two or three pores attached to separate DNA scaffolds, which can be recognized by conductance values that are 2 or 3 times higher than the unitary conductance. Experiments with scaffolds bearing fewer than 8 peptides showed a range of lower conductance levels, none of which were stable for more than 1 minute (Table S2 and Fig. S16).

In isolated cases, we observed the insertion of unstable pores with a greater conductance than the H-state, but not corresponding to $\mathrm{H}+\mathrm{L}$ or $\mathrm{H}+\mathrm{H}$ (Table $\mathrm{S} 2$ and Fig. S17). These unstable higher conductance states most likely originate from the formation of a pore with 9 or $10 \alpha$ helices that returns to a more stable octameric L- or H-configuration by expelling the supernumerary peptide chain(s). This suggests that rearrangements of the peptides after nanopore insertion are possible and that Wza octamers are not in a kinetically trapped state. In short, this Wza peptide variant has a clear propensity for assembly in planar lipid bilayers, but only the octameric assembly can form open pores that remain stable for extended periods.

\section{Loss of Wza nanopores from bilayer upon scaffold removal}

To highlight the importance of the DNA scaffold for nanopore stability, we prepared cleavable conjugates of the Wza peptide with all 12 oligos (Fig. 4a and Table S1). After assembly, the scaffolded Wza peptides could be inserted into planar lipid bilayers in the same way as the uncleavable constructs (Fig. 4b). As long as the peptides were attached to the scaffold the pore was stable and its conductance remained constant, and electrically indistinguishable from Wza pores on the uncleavable scaffold.

We then investigated the stability of open octameric Wza nanopores after separation of the scaffold by treatment with $10 \mathrm{mM}$ TCEP in the cis compartment. Within 2 minutes, we observed a complete loss of the pores, either through an intermediate L-state or through an unknown intermediate state that is almost fully closed (Fig. 4c). After the pore was lost, we 
a

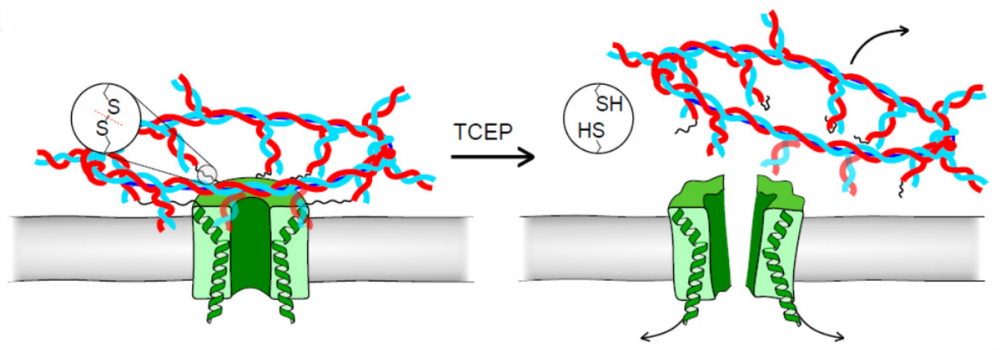

b
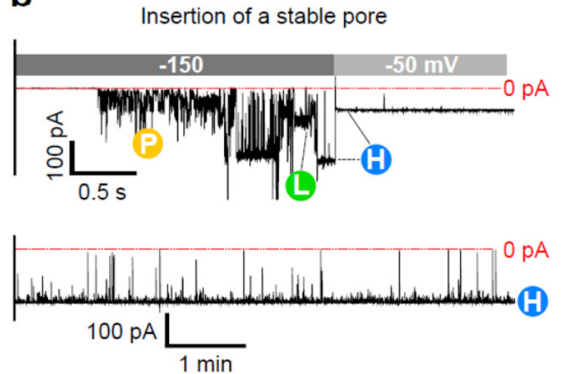

C

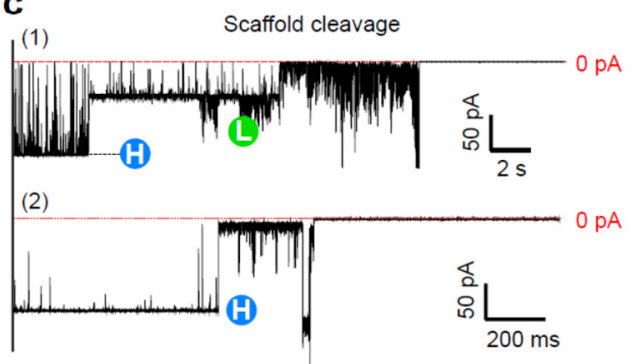

d

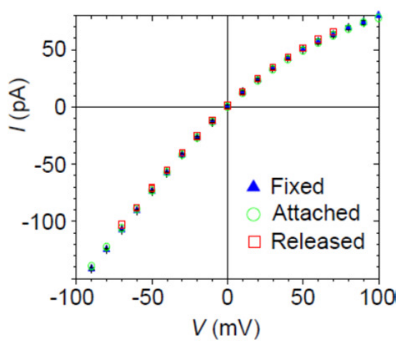

Figure 4 | The DNA scaffold stabilizes Wza nanopores.

a, Schematic illustration of the scaffold removal experiment. $\mathbf{b}$, Electrical recording of the insertion of a Wza nanopore formed from a DNA scaffold bearing 8 Wza peptides attached through cleavable disulfide linkers. The nanopore inserts through various precursor $(\mathrm{P})$ and $\mathrm{L}$ states before reaching the stable open-pore $\mathrm{H}$-state (top). The $\mathrm{H}$-state is stable for minutes at $-100 \mathrm{mV}$ when the scaffold is attached (bottom). c, Two exemplary electrical recordings of the loss of Wza nanopores at $-100 \mathrm{mV}$ after the DNA scaffold is cleaved off by the addition of $10 \mathrm{mM}$ TCEP (5 nanopores were tested). d, Current-voltage curves obtained from single Wza nanopores with a fixed scaffold (average of 3 nanopores) and with a cleavable scaffold before cleavage (average of 3 nanopores) and one experiment in which the scaffold was removed from a Wza pore by TCEP while the pore was kept at an applied potential of $+20 \mathrm{mV}$ to prevent loss. The current-voltage curve was recorded 13 minutes after TCEP addition.

observed no reinsertion, confirming our previous observation that free Wza peptides do not form stable nanopores in planar lipid bilayers. In one experiment, we managed to keep a Wza nanopore open by applying a low potential of $+20 \mathrm{mV}$ after the scaffold was cleaved. When the conductance of this unscaffolded pore was carefully examined, we found that it exhibited almost the same the conductance as scaffolded pores, with only a slightly less pronounced rectification (Fig. 4d). When the applied potential was stepped to $-90 \mathrm{mV}$, this unscaffolded pore was quickly lost via the L-state as well.

\section{The scaffold as a docking site}

Our DNA nanostructures are suited as docking stations for incoming target molecules that are linked to complementary oligos. This was investigated by using three example probes and a scaffold in which one of the arms was extended by up to 22 nucleotides to generate a docking site of adjustable affinity (Fig. 5a).

The scaffold with 8 covalently attached Wza peptides and a single docking site of unhybridized bases was assembled and added to a planar lipid bilayer. After the insertion of a single peptide nanopore and perfusion of the chamber, tagged probes were added to the cis side of the bilayer. When a PEG-5k polymer was bound to the 8-nucleotide tag, we observed 

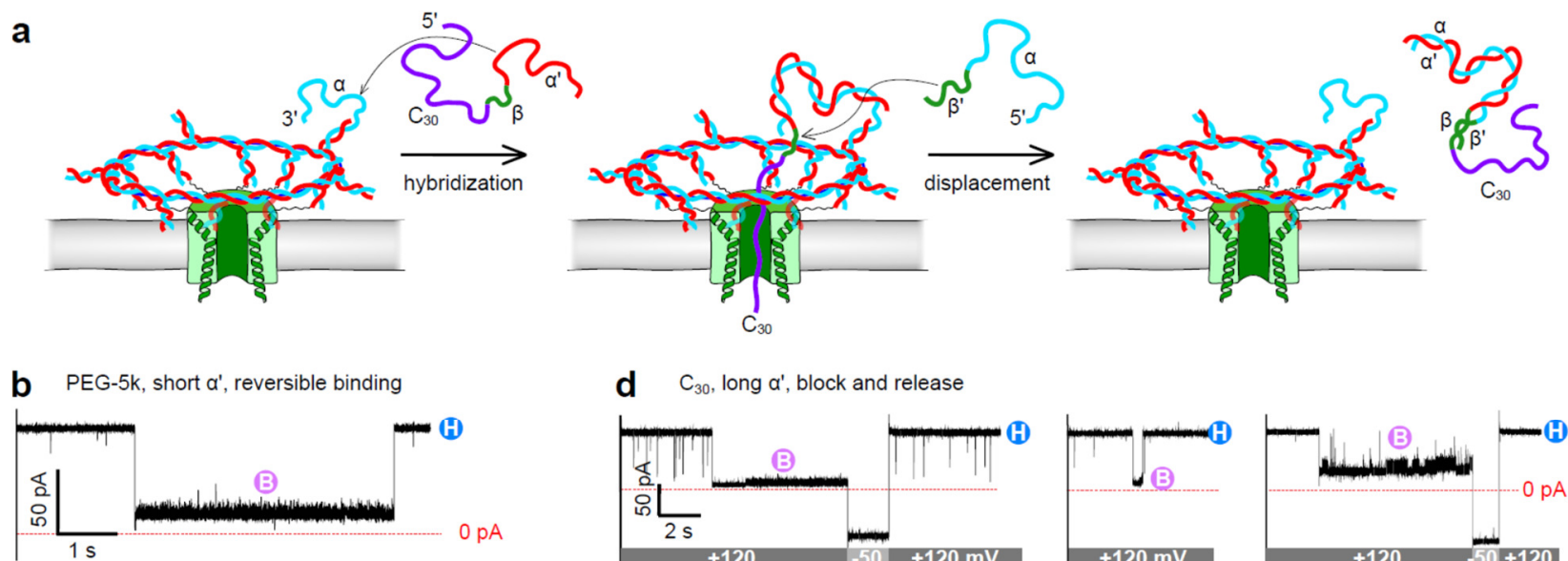

d $\quad C_{30}$, long $a^{\prime}$, block and release
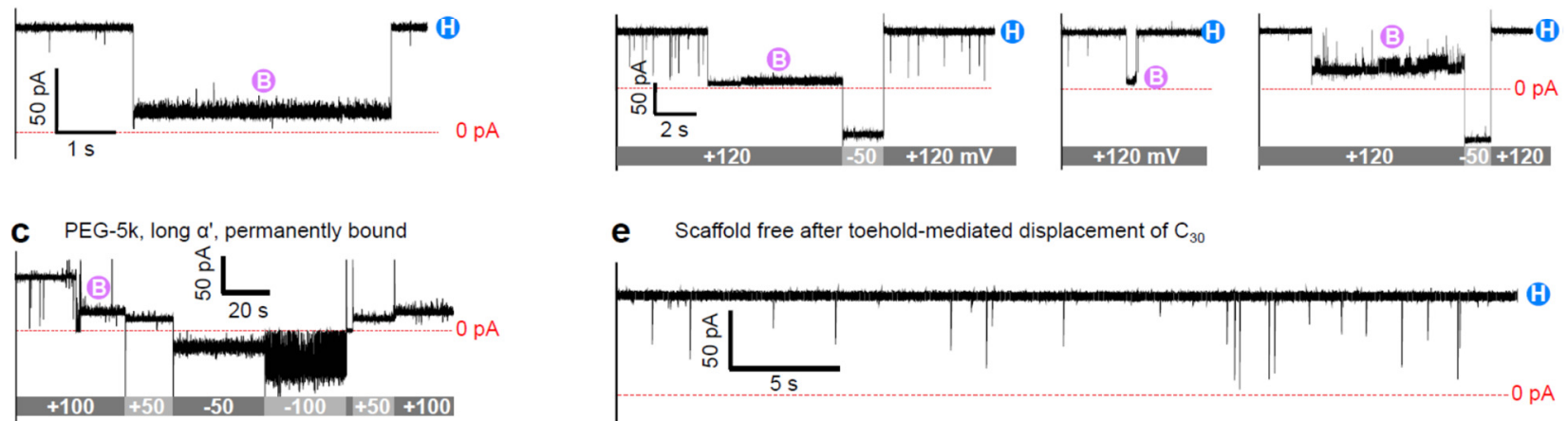

Figure 5 | The DNA scaffold is a versatile docking site for tagged oligonucleotides.

a, Schematic illustration of the docking and displacement of tagged oligonucleotides. $\mathbf{b}$, Electrical recording at +100 $\mathrm{mV}$ of the binding (B) and dissociation of PEG-5k attached to an 8-mer $\alpha^{\prime}$ oligo at (53 blockades were observed with 4 nanopores). c, Electrical recording of the binding and permanent blockade by PEG-5k attached to a 19-mer $\alpha^{\prime}$ oligo (2 nanopores). d, Three exemplary electrical recordings of the blockade by (B) and release from the nanopore of a poly $(\mathrm{dC})_{30}$ bound to the scaffold's $\alpha$-handle via an $\alpha^{\prime} \beta$ sequence (28 blockades with 3 nanopores). e, Electrical recording of the sustained $\mathrm{H}$-state of a scaffolded Wza nanopore after removal of the bound poly $(\mathrm{dC})_{30}$ by a toeholdmediated strand displacement with a 32-mer $\alpha \beta^{\prime}$ oligo at $+120 \mathrm{mV}$.

reversible binding, as evidenced by transient current blockades with a mean duration of $466 \pm 14$ $\mathrm{ms}$ and an average residual current of $15 \pm 1.2 \%$ (Fig. 5b, Figs. S18-S19 and Table S3). The short 8-mer duplexes have a limited lifetime, previously found to range from 0.1-1.0 s, depending on the sequence of the oligo ${ }^{33}$.

PEG binding can be made essentially irreversible with a longer hybridization sequence of 19 nucleotides. In this case, instead of transient blocking events, we observed permanent blockades with a residual current of $18 \%$ that could not be reversed by varying the applied potential between -100 and $+100 \mathrm{mV}$ (Fig. 5c). Eventually, these measurements concluded by loss of the pore, for example by rupture of the bilayer.

When a $\mathrm{C}_{30}$ oligo tag was attached to the same 19-mer handle, the blocking characteristics changed. As the $\mathrm{C}_{30}$ end entered the pore, the current was reduced to $12 \pm 5 \%$ (cf. Fig. $3 \mathrm{~g}$ ). The tagged oligo could not translocate through the pore because it was hybridized to the scaffold, hence the blockade persisted in most cases (Fig. 5d). Reversal of the applied potential resulted in release of oligo from the constriction and unblocking of the pore. The $\mathrm{C}_{30}$-tagged oligo could be fully removed from the scaffold through toehold-mediated strand displacement, in which a 
second oligo was hybridized to a toehold region ( $\beta$, Fig. 5a) between the $\mathrm{C}_{30}$ and the hybridized handle. We observed no more blocking events after the addition of a 1.5-fold excess of the displacement strand $\left(\alpha \beta^{\prime}\right)$, establishing that the docking site on the scaffold had been freed (Fig. $5 e)$.

\section{Pre-organization of pore-forming peptides on DNA scaffolds}

Synthetic supramolecular scaffolds have not been used previously to guide the assembly of transmembrane peptide assemblies. In our ring-shaped DNA scaffold all oligos were modified with peptides and purified before assembly, providing confidence in the final stoichiometry of the scaffolded nanostructure. The inevitable non-quantitative nature of conjugation reactions on preformed scaffolds and the presence of excess free oligos or peptide is of no concern here, as all of the peptide-oligo conjugates were purified and analysed separately. By choosing a scaffold that closes only when all the constituent short oligonucleotides are present, instead of hybridizing multiple short oligos to a long scaffold strand, as is common for DNA origami ${ }^{34}$, we avoided the possibility that a fraction of short oligos could inadvertently be excluded from the scaffold. The implementation of our strategy indeed showed that the number of scaffolded peptides in the construct can be precisely tuned without compromising the scaffold integrity (Fig. 2c), although this does not imply that all scaffolded constructs are able to form functional nanopores with the same number of peptides as templated. Based on the measured purities of the individual conjugates (Table S1), we infer that $96 \%$ of the scaffolds that were designed to bear 8 peptides actually hold the anticipated number of 8 peptides.

The DNA scaffold lowered the concentration of peptides required for pore formation significantly by preconcentrating the peptides on the scaffold: 150-400 nM Wza peptide was added for control experiments (Figs. S7-S13), while only 5-40 nM of scaffolded peptides was used (Figs. 3-5). However, despite preconcentration of up to 12 peptides on a single scaffold, we observed the insertion of nanopores of the same conductance for all scaffolds with at least 8 peptides (Table S2), suggesting that the $\alpha$-helices that compose the Wza D4 domain preferentially form octameric barrels, possibly because the D4 domain has evolved so that the octameric configuration is of the lowest free energy. We therefore suppose that it is not possible to force another stoichiometry upon Wza peptides simply by bringing them together on the DNA scaffold used here. However, it might be possible to change the stoichiometry of nanopores made of more promiscuous peptides, or proteins that are known to form multiple species naturally, such as ClyA $^{35}$, with our DNA scaffold. To change the preferential stoichiometry of a homomeric nanopore, such as Wza, a more rigid scaffold that fits only one state could be used ${ }^{12}$, but this will 
come at the expense of scaffold versatility: a specific scaffold will be required for every peptide and every stoichiometry.

Our demonstration that DNA scaffolds can be used to preconcentrate peptides and stabilize their assemblies to form membrane-spanning pores is of importance in several areas. For many synthetic peptides designed to form transmembrane channels and pores, it is difficult to obtain a crystal structure in order to determine the stoichiometry, pin down the transmembrane region and discern the orientation within the membrane ${ }^{12,36-40}$. The present approach is a direct means to control the stoichiometry, or at least the upper limit, and the direction of insertion of candidate peptides into bilayers, provided the DNA scaffold does not restrict their assembly. For example, we attached the Wza peptides through their $\mathrm{C}$ termini to the DNA scaffold, leaving the $\mathrm{N}$ termini free. We did not observe insertions even at a tenfold higher concentration than that used for the N-terminus-attached Wza peptides (Fig. S20), confirming that Wza requires a free C terminus for insertion into planar lipid bilayers ${ }^{9,10,12}$.

\section{Conclusions}

DNA rings can serve as scaffolds to template the formation of amphiphilic $\alpha$-helical Wza peptides into stable octameric nanopores of uniform conductance. The scaffold is essential for the stability of these pores and can be used to bind a variety of tagged oligos (with or without cargoes) after bilayer insertion. To enable the formation of uniform nanopores with various numbers of subunits, our scaffolds might also be used to direct the assembly of more promiscuous peptides, either designed de novo ${ }^{41}$ or derived from antimicrobial peptides ${ }^{28}$. The ability to form uniform large nanopores would open an immense range of possibilities, ranging from the detection and fingerprinting of proteins in complex mixtures ${ }^{42,43}$ to single-molecule studies of enzymes ${ }^{44}$ and the mapping of epigenetic markers on long strands of dsDNA ${ }^{45}$. DNA scaffolds could further be used to form well-defined assemblies of multiple membrane proteins and pore ${ }^{20}$, each held on a different arm or on separate linked scaffolds. To prevent degradation of these scaffolds in living cells, one could use synthetic analogues of DNA, such as $\mathrm{XNA}^{46}$. Finally, the control over the relative position of each oligo in our scaffold will allow the assembly of well-known nanopores, such as $\alpha$-haemolysin, with specified permutations of the subunits around a central axis, as a first step towards creating artificial enzymes ${ }^{47,48}$. 


\section{References}

1. Jentsch, T. J., Hübner, C. A. \& Fuhrmann, J. C. Ion channels: Function unravelled by dysfunction. Nature Cell Biol. 6, 1039-1047 (2004).

2. Corry, B. \& Chung, S. H. Mechanisms of valence selectivity in biological ion channels. Chem. Mol. Life Sci. 63, 301-315 (2006).

3. Gilbert, R. J. C., Serra, M. D., Froelich, C. J., Wallace, M. I. \& Anderluh, G. Membrane pore formation at protein-lipid interfaces. Trends Biochem. Sci. 39, 510-516 (2014).

4. Branton, D., Deamer, D. W., Marziali, A., Bayley, H., Benner, S. A., Butler, T., et al. The potential and challenges of nanopore sequencing. Nature Biotechnol. 26, 1146-1153 (2008).

5. Ayub, M. \& Bayley, H. Engineered transmembrane pores. Curr. Opin. Chem. Biol. 34, 117-126 (2016).

6. Koebnik, R., Locher, K. P. \& Van Gelder, P. Structure and function of bacterial outer membrane proteins: barrels in a nutshell. Mol. Microbiol. 37, 239-253 (2000).

7. Tamm, L. K., Hong, H. \& Liang, B. Folding and assembly of $\beta$-barrel membrane proteins. Biochim. Biophys. Acta 1666, 250-263 (2004).

8. Gu, Y., Li, H., Dong, H., Zeng, Y., Zhang, Z., Paterson, N. G., et al. Structural basis of outer membrane protein insertion by the BAM complex. Nature 531, 64-69 (2016).

9. Dong, C., Beis, K., Nesper, J., Brunkan-LaMontagne, A. L., Clarke, B. R., Whitfield, C., et al. Wza the translocon for E. coli capsular polysaccharides defines a new class of membrane protein. Nature 444, 226229 (2006).

10. Kong, L., Harrington, L., Li, Q., Cheley, S., Davis, B. G. \& Bayley, H. Single-molecule interrogation of a bacterial sugar transporter allows the discovery of an extracellular inhibitor. Nature Chem. 5, 651-659 (2013).

11. Soskine, M., Biesemans, A., Moeyaert, B., Cheley, S., Bayley, H. \& Maglia, G. An Engineered ClyA Nanopore Detects Folded Target Proteins by Selective External Association and Pore Entry. Nano Letters 12, 4895-4900 (2012).

12. Mahendran, K. R., Niitsu, A., Kong, L., Thomson, A. R., Sessions, R. B., Woolfson, D. N., et al. A monodisperse transmembrane $\alpha$-helical peptide barrel. Nature Chem. 9, 411-419 (2017).

13. Mutter, M. \& Vuilleumier, S. A Chemical Approach to Protein Design — Template-Assembled Synthetic Proteins (TASP). Angew. Chem. Int. Ed. 28, 535-554 (1989).

14. Futaki, S. Peptide ion channels: Design and creation of function. Pept. Sci. 47, 75-81 (1998).

15. Bayley, H. \& Jayasinghe, L. Functional engineered channels and pores (Review). Mol. Membrane Biol. 21, 209-220 (2004).

16. Pinheiro, A. V., Han, D., Shih, W. M. \& Yan, H. Challenges and opportunities for structural DNA nanotechnology. Nature Nanotechnol. 6, 763-772 (2011).

17. Wilner, O. I., Shimron, S., Weizmann, Y., Wang, Z.-G. \& Willner, I. Self-Assembly of Enzymes on DNA Scaffolds: En Route to Biocatalytic Cascades and the Synthesis of Metallic Nanowires. Nano Letters 9 , 2040-2043 (2009).

18. Fu, J., Yang, Y. R., Johnson-Buck, A., Liu, M., Liu, Y., Walter, N. G., et al. Multi-enzyme complexes on DNA scaffolds capable of substrate channelling with an artificial swinging arm. Nature Nanotechnol. 9, 531-536 (2014).

19. Zhang, Y., Tsitkov, S. \& Hess, H. Proximity does not contribute to activity enhancement in the glucose oxidase-horseradish peroxidase cascade. Nature Commun. 7, 13982 (2016).

20. Raschle, T., Lin, C., Jungmann, R., Shih, W. M. \& Wagner, G. Controlled Co-reconstitution of Multiple Membrane Proteins in Lipid Bilayer Nanodiscs Using DNA as a Scaffold. ACS Chem. Biol. 10, 2448-2454 (2015).

21. Lee, H., Lytton-Jean, A. K. R., Chen, Y., Love, K. T., Park, A. I., Karagiannis, E. D., et al. Molecularly self-assembled nucleic acid nanoparticles for targeted in vivo siRNA delivery. Nature Nanotechnol. 7, 389393 (2012).

22. Douglas, S. M., Bachelet, I. \& Church, G. M. A Logic-Gated Nanorobot for Targeted Transport of Molecular Payloads. Science 335, 831-834 (2012).

23. Langecker, M., Arnaut, V., Martin, T. G., List, J., Renner, S., Mayer, M., et al. Synthetic Lipid Membrane Channels Formed by Designed DNA Nanostructures. Science 338, 932-936 (2012).

24. Burns, J. R., Stulz, E. \& Howorka, S. Self-Assembled DNA Nanopores That Span Lipid Bilayers. Nano Letters 13, 2351-2356 (2013).

25. Karshikoff, A., Nilsson, L. \& Ladenstein, R. Rigidity versus flexibility: the dilemma of understanding protein thermal stability. FEBS J. 282, 3899-3917 (2015).

26. von Krbek, L. K. S., Achazi, A. J., Schoder, S., Gaedke, M., Biberger, T., Paulus, B., et al. The Delicate Balance of Preorganisation and Adaptability in Multiply Bonded Host-Guest Complexes. Chem. Eur. J. 23, 2877-2883 (2017). 
27. Jolliffe, K. A. Backbone-modified Cyclic Peptides: New Scaffolds for Supramolecular Chemistry. Supramolecular Chemistry 17, 81-86 (2005).

28. Song, C., Weichbrodt, C., Salnikov, E. S., Dynowski, M., Forsberg, B. O., Bechinger, B., et al. Crystal structure and functional mechanism of a human antimicrobial membrane channel. Proc. Natl Acad. Sci. USA 110, 4586-4591 (2013).

29. Bowie, J. U. Solving the membrane protein folding problem. Nature 438, 581-589 (2005).

30. Krasilnikov, O. V., Merzlyak, P. G., Yuldasheva, L. N., Rodrigues, C. G., Bhakdi, S. \& Valeva, A. Electrophysiological evidence for heptameric stoichiometry of ion channels formed by Staphylococcus aureus alpha-toxin in planar lipid bilayers. Mol. Microbiol. 37, 1372-1378 (2000).

31. Salay, L. C., Procopio, J., Oliveira, E., Nakaie, C. R. \& Schreier, S. Ion channel-like activity of the antimicrobial peptide tritrpticin in planar lipid bilayers. FEBS Lett. 565, 171-175 (2004).

32. Paulmann, M., Arnold, T., Linke, D., Özdirekcan, S., Kopp, A., Gutsmann, T., et al. Structure-Activity Analysis of the Dermcidin-derived Peptide DCD-1L, an Anionic Antimicrobial Peptide Present in Human Sweat. J. Biol. Chem. 287, 8434-8443 (2012).

33. Howorka, S. \& Bayley, H. Probing Distance and Electrical Potential within a Protein Pore with Tethered DNA. Biophys. J. 83, 3202-3210 (2002).

34. Rothemund, P. W. K. Folding DNA to create nanoscale shapes and patterns. Nature 440, 297-302 (2006).

35. Soskine, M., Biesemans, A., De Maeyer, M. \& Maglia, G. Tuning the Size and Properties of ClyA Nanopores Assisted by Directed Evolution. J. Am. Chem. Soc. 135, 13456-13463 (2013).

36. Nicol, F., Nir, S. \& Szoka, F. C. Orientation of the Pore-Forming Peptide GALA in POPC Vesicles Determined by a BODIPY-Avidin/Biotin Binding Assay. Biophys. J. 76, 2121-2141 (1999).

37. Li, W., Nicol, F. \& Szoka, F. C. GALA: a designed synthetic pH-responsive amphipathic peptide with applications in drug and gene delivery. Adv. Drug Deliv. Rev. 56, 967-985 (2004).

38. Rausch, J. M., Marks, J. R. \& Wimley, W. C. Rational combinatorial design of pore-forming $\beta$-sheet peptides. Proc. Natl Acad. Sci. USA 102, 10511-10515 (2005).

39. Krauson, A. J., He, J., Wimley, A. W., Hoffmann, A. R. \& Wimley, W. C. Synthetic Molecular Evolution of Pore-Forming Peptides by Iterative Combinatorial Library Screening. ACS Chem. Biol. 8, 823-831 (2013).

40. Gupta, K., Singh, S. \& van Hoek, L. M. Short, Synthetic Cationic Peptides Have Antibacterial Activity against Mycobacterium smegmatis by Forming Pores in Membrane and Synergizing with Antibiotics. Antibiotics 4, 358-378 (2015).

41. Thomson, A. R., Wood, C. W., Burton, A. J., Bartlett, G. J., Sessions, R. B., Brady, R. L., et al. Computational design of water-soluble $\alpha$-helical barrels. Science 346, 485 (2014).

42. Yusko, E. C., Bruhn, B. R., Eggenberger, O. M., Houghtaling, J., Rollings, R. C., Walsh, N. C., et al. Realtime shape approximation and fingerprinting of single proteins using a nanopore. Nature Nanotechnol. 12, 360-367 (2017).

43. Huang, G., Willems, K., Soskine, M., Wloka, C. \& Maglia, G. Electro-osmotic capture and ionic discrimination of peptide and protein biomarkers with FraC nanopores. Nature Commun. 8, 935 (2017).

44. Soskine, M., Biesemans, A. \& Maglia, G. Single-Molecule Analyte Recognition with ClyA Nanopores Equipped with Internal Protein Adaptors. J. Am. Chem. Soc. 137, 5793-5797 (2015).

45. Wanunu, M., Cohen-Karni, D., Johnson, R. R., Fields, L., Benner, J., Peterman, N., et al. Discrimination of Methylcytosine from Hydroxymethylcytosine in DNA Molecules. J. Am. Chem. Soc. 133, 486-492 (2011).

46. Taylor, A. I., Beuron, F., Peak-Chew, S.-Y., Morris, E. P., Herdewijn, P. \& Holliger, P. Nanostructures from Synthetic Genetic Polymers. ChemBioChem 17, 1107-1110 (2016).

47. Hammerstein, A. F., Jayasinghe, L. \& Bayley, H. Subunit Dimers of $\alpha$-Hemolysin Expand the Engineering Toolbox for Protein Nanopores. J. Biol. Chem. 286, 14324-14334 (2011).

48. Lee, J., Boersma, A. J., Boudreau, M. A., Cheley, S., Daltrop, O., Li, J., et al. Semisynthetic Nanoreactor for Reversible Single-Molecule Covalent Chemistry. ACS Nano 10, 8843-8850 (2016).

\section{Acknowledgements}

E.S. acknowledges support from the European Union's Horizon 2020 Programme under grant 655660 (Hybripore). The work was supported by the BBSRC and a European Research Council Advanced Grant. The authors thank Dr. Ofer Wilner and Dr. Kozhinjampara R. Mahendran for their helpful discussions, Dr. Errin Johnson for help with the TEM measurements and Dr. Idlir Liko and Prof. Carol V. Robinson for their assistance in mass spectrometry. 


\section{Author contributions}

E.S. and H.B. conceived and designed experiments and wrote the paper. E.S. performed experiments and simulations and analysed data. S.T. performed photobleaching experiments and analysed data. All authors discussed and commented on the manuscript.

\section{Data availability}

The data that support the plots within this paper and other findings of this study are available from the corresponding author upon reasonable request

\section{Supplementary information}

Supplementary information is available in the online version of the paper. Reprints and permission information is available online at www.nature.com/reprints. Correspondence and requests for materials should be addressed to $\mathrm{H}$.

Bayley or E. Spruijt. 


\section{Methods}

\section{Materials}

Oligonucleotides were purchased from Biomers (Ulm, Germany) or Sigma Aldrich (Haverhill, UK) as HPLCpurified, dried samples. All Wza peptides were purchased from Peptide Protein Research Ltd (Fareham, UK) at $>95 \%$ purity (HPLC) as lyophilized powders. Precast gels were purchased from Bio-Rad (Watford, UK), lipids from Avanti Polar Lipids (Alabaster, AL) and all other chemicals from Sigma Aldrich, unless otherwise stated.

\section{Peptide-oligonucleotide conjugation}

The preparation of peptide-oligonucleotide conjugates was based in part on the procedure reported by Williams and Chaput $^{49}$. Briefly, $10 \mathrm{nmol}(20 \mu \mathrm{L}, 500 \mu \mathrm{M}) 5^{\prime}$-amino-C6-functionalized oligonucleotide was mixed with $60 \mu \mathrm{L} 100$ $\mathrm{mM}$ potassium phosphate, $\mathrm{pH} 7.2$, and $500 \mathrm{nmol}$ of a bifunctional linker, and incubated for $2 \mathrm{~h}$ at room temperature. For uncleavable peptide-oligo conjugates, $10 \mathrm{mg} / \mathrm{mL}$ succinimidyl-([N-maleimidopropionamido]-diethylene glycol) ester ( $\mathrm{SMPEG}_{2}$, from Thermo Fisher) in DMSO was used. For cleavable conjugates, $50 \mathrm{mg} / \mathrm{mL}$ 2-pyridyldithiotetraoxatetradecane-N-hydroxysuccinimide ( $\mathrm{PEG}_{4}$-SPDP, from Thermo Fisher) in DMSO was used. After incubation, $100 \mathrm{mM}$ potassium phosphate was added to the reaction mixture to reach a total volume of $140 \mu \mathrm{L}$. Removal of excess reagent and transfer into $100 \mathrm{mM}$ potassium phosphate, $\mathrm{pH} 7.2$, was done by three desalting steps with PD SpinTrap G25 columns (GE Healthcare). Electrospray ionization mass spectrometry (ESI-MS) revealed a linker attachment yield of $>90 \%$ after this step, with about $10 \%$ hydrolysis of the maleimide group in case of $\mathrm{SMPEG}_{2}$.

Meanwhile, the Wza peptide was dissolved in $100 \mathrm{mM}$ potassium phosphate, $\mathrm{pH} 7.2$, with $0.01 \%(\mathrm{w} / \mathrm{w}) n$ dodecyl- $\beta$ - $D$-maltoside (DDM, from Thermo Fisher) to a typical concentration of $0.15 \mathrm{mg} / \mathrm{mL}$. The dissolved peptide was reduced by incubation with immobilized-TCEP disulfide-reducing agarose gel beads (Pierce/Thermo Fisher). After removal of the beads, the concentration of the reduced peptide was measured with a NanoDrop spectrometer. Reduced peptide ( $20 \mathrm{nmol})$ was added to the triply desalted oligo-linker solution, and the reaction mixture was incubated at room temperature for $12 \mathrm{~h}$.

The conjugates were purified by HPLC (Agilent Infinity 1260 series) by using a $\mathrm{C}_{18}$ column ( $4.6 \times 150$ $\mathrm{mm}$ ), with $10 \mathrm{mM}$ tetraethylammonium bromide (TEAB) in MilliQ water, $\mathrm{pH}$ 8.5, as the binding buffer (A), and acetonitrile for elution (buffer B). Oligos were bound to the column in $96 \%$ A, and eluted by using a gradient from $4 \%$ to $65 \%$ B over 10 minutes, at $1 \mathrm{~mL} / \mathrm{min}$. Unmodified oligos were eluted at $30 \% \mathrm{~B}$, and Wza-modified oligos at $45 \%$ B. The peptide-modified oligos were lyophilized, dissolved in $50 \mu \mathrm{L} 100 \mathrm{mM}$ potassium phosphate, $\mathrm{pH} 7.2$, diluted to $5 \mu \mathrm{M}$, and stored at $-80^{\circ} \mathrm{C}$ until use.

\section{Annealing of the DNA scaffold with attached Wza peptides}

Equal amounts of the 12 oligonucleotides (see Supplementary Data 1 for sequence details) were mixed at an overall strand concentration of $200 \mathrm{nM}$ in a total volume of $50 \mu \mathrm{L}$, containing $100 \mathrm{mM}$ potassium phosphate, $\mathrm{pH}$ 7.2, $0.5 \mathrm{M}$ $\mathrm{NaCl}$, and $0.02 \% \mathrm{w} / \mathrm{w}$ DDM for the peptide-modified oligos. The oligos were then annealed in a Veriti Thermal Cycler (Applied Biosystems) by heating the sample for $3 \mathrm{~min}$ at $95^{\circ} \mathrm{C}$, cooling to $65^{\circ} \mathrm{C}$ in $3 \mathrm{~min}$ and finally cooling to $4^{\circ} \mathrm{C}$ in $4 \mathrm{~h}$. The samples were stored at $4^{\circ} \mathrm{C}$ for up to one week until use. 


\section{Electrical recordings}

A planar lipid bilayer of 1,2-diphytanoyl-sn-glycero-3-phosphocholine (DPhPC) was formed across an aperture of $100 \mu \mathrm{m}$ diameter in a teflon film sandwiched between two polycarbonate half cells with a compartment volume of $0.5 \mathrm{~mL}$. The teflon film was prepainted with a solution of hexadecane in $n$-pentane $(1 \% \mathrm{w} / \mathrm{w})$. The assembled chamber was placed in a Faraday cage on an antivibration table. An electrical circuit was then formed by a pair of $\mathrm{Ag} / \mathrm{AgCl}$ electrodes set in 1.5\% agarose in $3 \mathrm{M} \mathrm{KCl}$, connected to an Axopatch 200B amplifier and a Digidata 1440A A/D converter. The electrode in the cis compartment was grounded, while the electrode in the trans compartment was used as the working electrode. The compartments were filled with electrolyte solution (10 mM HEPES, pH 7.4, 1 M $\mathrm{KCl})$ and $\mathrm{DPhPC}$ in $n$-pentane $(3 \mu \mathrm{L}, 5 \mathrm{mg} / \mathrm{mL})$ was added to each side. After evaporation of the $n$-pentane, bilayers were formed by using the Montal-Mueller technique ${ }^{50}$. Peptide-bearing DNA scaffolds (1-4 nM) or scaffold-free peptides (150-400 $\mathrm{nM}$ ) were added to the cis compartment and the insertion of pores into the bilayer was observed under an applied potential of -100 or $-150 \mathrm{mV}$. Data were recorded using pClamp 10.3 (Molecular Devices) with a low-pass filter frequency of $2 \mathrm{kHz}$ and a sampling frequency of $10 \mathrm{kHz}$, unless stated otherwise. The data were analysed and prepared for presentation with Clampfit.

\section{References}

49. Williams, B. A. R. \& Chaput, J. C. Synthesis of Peptide-Oligonucleotide Conjugates Using a Heterobifunctional Crosslinker. Current Protocols in Nucleic Acid Chemistry. John Wiley \& Sons, Inc., 2001.

50. Gutsmann, T., Heimburg, T., Keyser, U., Mahendran, K. R. \& Winterhalter, M. Protein reconstitution into freestanding planar lipid membranes for electrophysiological characterization. Nature Protoc. 10, 188-198 (2015). 